\title{
Influence of Organic and Conventional Farming on Grain Yield and Protein Composition of Chickpea Genotypes
}

\author{
Michele Andrea De Santis ${ }^{1}\left({ }^{(}\right.$, Michele Rinaldi $^{2}{ }^{(}$,, Valeria Menga ${ }^{2}$, Pasquale Codianni ${ }^{2}$, Luigia Giuzio ${ }^{1}$, \\ Clara Fares ${ }^{2, *(D)}$ and Zina Flagella ${ }^{1, *}$ \\ 1 Department of Agriculture, Food, Natural resources and Engineering (DAFNE), University of Foggia, \\ 71122 Foggia, Italy; michele.desantis@unifg.it (M.A.D.S.); luigia.giuzio@unifg.it (L.G.) \\ 2 CREA Research Centre for Cereal and Industrial Crops, 71121 Foggia, Italy; \\ michele.rinaldi@crea.gov.it (M.R.); valeria.menga@crea.gov.it (V.M.); pasquale.codianni@crea.gov.it (P.C.) \\ * Correspondence: clara.fares@crea.gov.it (C.F.); zina.flagella@unifg.it (Z.F.); Tel.: +39-0881589360 (Z.F.)
}

Citation: De Santis, M.A.; Rinaldi, M.; Menga, V.; Codianni, P.; Giuzio, L.; Fares, C.; Flagella, Z. Influence of Organic and Conventional Farming on Grain Yield and Protein Composition of Chickpea Genotypes. Agronomy 2021, 11, 191. https://doi.org/ 10.3390/agronomy11020191

Academic Editors: Enrico Francia and Domenico Ronga

Received: 7 December 2020

Accepted: 17 January 2021

Published: 20 January 202

Publisher's Note: MDPI stays neutral with regard to jurisdictional claims in published maps and institutional affiliations.

Copyright: (C) 2021 by the authors. Licensee MDPI, Basel, Switzerland. This article is an open access article distributed under the terms and conditions of the Creative Commons Attribution (CC BY) license (https:// creativecommons.org/licenses/by/ $4.0 /)$.

\begin{abstract}
Chickpea is a key crop in sustainable cropping systems and for its nutritional value. Studies on agronomic and genetic influences on chickpea protein composition are missing. In order to obtain a deep insight into the genetic response of chickpeas to management in relation to agronomic and quality traits, a two-year field trial was carried out with eight chickpea genotypes under an organic and conventional cropping system. Protein composition was assessed by SDS-PAGE in relation to the main fractions (vicilin, convicilin, legumin, lectin, 2s-albumin). Crop response was highly influenced by year and presumably also by management, with a $-50 \%$ decrease in grain yield under organic farming, mainly due to a reduction in seed number per $\mathrm{m}^{2}$. No effect of crop management was observed on protein content, despite significant differences in terms of protein composition. The ratio between the major globulins, $7 \mathrm{~s}$ vicilin and 11s legumin, showed a negative relationship with grain yield and was found to be higher under organic farming. Among genotypes, black-seed Nero Senise was characterized by the highest productivity and water-holding capacity, associated with low lectin content. These findings highlight the importance of the choice of chickpea genotypes for cultivation under organic farming in relation to both agronomic performance and technological and health quality.
\end{abstract}

Keywords: chickpea; organic farming; legume proteins; vicilin; legumin; lectin; pulse quality

\section{Introduction}

Legumes are considered the second most important source of human food after cereals, with pulses including only those species belonging to the legume family and whose product is represented by dry grain to be used as it is, according to the FAO classification [1] Legumes have a key role in crop rotation, especially for their nitrogen-fixing ability, which is particularly advantageous for cereals [2]. Grain legumes are a valuable source of food proteins, but also of other nutrients such as starch, dietary fiber, vitamins, minerals and phenols [3]. In order to encourage political actions to promote the cultivation of these valuable crops, the FAO declared 2016 to be "the International year of Pulses" (ONU A/RES/68/231).

Chickpea (Cicer arietinum L.) is the third most important legume crop in the world, following the common bean and the pea, and one of the major ones cultivated in the Mediterranean basin, with an increasing trend of cultivation also in Italy [1].

Chickpea is one of the earliest cultivated vegetables, starting around 7450 years ago in the Middle East [4]. There are two main commercially available types of chickpea grown in the world: desi and kabuli seed types. The former has a small seed with a dark irregular-shaped seed coat and is grown on semi-arid land, especially Asia and Africa, while the latter is larger than the desi chickpea, has a thin light-colored seed coat and is normally grown in temperate regions of the world, including Europe, North Africa, West 
Asia, North America and Australia [5]. The chickpea is also well adapted to temperate environments, such as the Mediterranean basin, due to its tolerance to moderate water deficit. Its main limitation is represented by ascochyta blight (AB) caused by Ascochyta rabiei Pass., which can lead to severe loss of grain production [6].

Chickpea seed is generally rich in carbohydrates (54-71\%), including starch and dietary fiber, physiological active compounds and good-quality protein (18-26\%). Proteins are stored in membrane-bound organelles (protein bodies) and are a store of amino acids for developing seedlings. As for other legumes, chickpea proteins are classified according to their solubility into albumins (soluble in water; 10-14\%), globulins (soluble in salt solutions; $55-60 \%$ ), prolamins (hydro-alcoholic solutions; 2-3\%) and glutelin (acid/alkali solutions; $18 \%$ ). Globulins are composed of the two major groups, classified on the basis of the sedimentation index into $11 \mathrm{~s}$ legumin and $7 \mathrm{~s}$ vicilin and convicilin, which belong to the cupin superfamily. Within albumin fraction, the major component is represented by $2 \mathrm{~s}$ albumin, which consist of two subunits of $8-10 \mathrm{kDa}$ and $4-5 \mathrm{kDa}$, linked by two disulfide bounds. This fraction is rich in cysteine; meanwhile, chickpea proteins are generally poor in sulfur amino acids. Other minor proteins include lectins, protease inhibitors and lipoxygenase [3,7].

In recent years, research activities have been focused on the exploration and characterization of chickpea germplasm, in order to pursue breeding goals of biotic (AB) and abiotic resistance and nutritional quality [8-10]. Furthermore, the increasing interest in spreading pulses in crop systems is leading to the development of agronomic and crop physiology studies in order to promote environmental and economic sustainability [11-13]. Experimental studies on the improvement of chickpea productivity and food quality under organic farming are necessary to achieve sustainable goals. Nowadays, little information is available on this topic, in particular with respect to the effect on health quality. However, preliminary studies seem encouraging $[14,15]$.

The aim of the current study was to explore genetic variability in terms of response to different farming systems; in order to do this, eight different chickpea genotypes were cultivated under organic and conventional management and both agronomic and quality performances were evaluated in relation to the changes in protein composition, assessed by electrophoresis analysis.

\section{Materials and Methods}

\subsection{Field Experiments}

Two experimental field trials, under conventional and organic management, were carried out at Foggia at the experimental farm of the Council for Agricultural Research and Economics, CREA-CI, Foggia $\left(41^{\circ} 27^{\prime} 03^{\prime \prime} \mathrm{N}, 15^{\circ} 30^{\prime} 06^{\prime \prime} \mathrm{E}\right)$, during two consecutive crop years (2013/14 and 2014/15, referred to as 2014 and 2015, respectively). The genetic material adopted in the experiments included eight different chickpea (Cicer arietinum L.) genotypes selected within Italian germplasm. The seed characteristics are detailed in Table 1.

Table 1. Seed characteristics of investigated chickpea (Cicer arietinum L.) genotypes.

\begin{tabular}{cccc}
\hline Genotype & Seed Type & Seed Color & Seed Size \\
\hline Calia & Rough & Yellow & Medium \\
Kairo & Rough & Yellow & Medium \\
Nero Senise & Rough & Black & Small \\
Pascià & Rough & Yellow & Large \\
Principe & Rough & Yellow & Large \\
Reale & Smooth & Yellow & Large \\
Sultano & Smooth & Yellow & Medium \\
Vulcano & Smooth & Yellow & Medium \\
\hline
\end{tabular}

A complete randomized block design was used, with three replicates, within organic and conventional cropping systems (OCS and CCS, respectively). The OCS was based 
at a field that had been managed under organic farming for the previous 14 years and no chemical inputs had been used as fertilizers or for weed control. The experimental plots were seeded on a field that was left fallow during the previous year. The soil used under the organic farming system was a clay loam (Typic Chromoxerert) with the following physical and chemical characteristics: $36.9 \mathrm{~g} \mathrm{~kg}^{-1}$ clay, $50.5 \mathrm{~g} \mathrm{~kg}^{-1}$ silt, $12.5 \mathrm{~g} \mathrm{~kg}^{-1}$ sand, $15 \mathrm{mg} \mathrm{kg}^{-1}$ available phosphorus (Olsen method), $800 \mathrm{mg} \mathrm{kg}^{-1}$ exchangeable potassium, $1.4 \mathrm{~g} \mathrm{~kg}^{-1}$ total $\mathrm{N}$ and $21 \mathrm{~g} \mathrm{~kg}^{-1}$ organic matter, $\mathrm{pH} 8$. An adjacent silt loam soil (Typic Chromoxerert) was adopted for the CCS experiment, with the following physical and chemical characteristics: $15.9 \mathrm{~g} \mathrm{~kg}^{-1}$ clay, $48.7 \mathrm{~g} \mathrm{~kg}^{-1}$ silt, $35.4 \mathrm{~g} \mathrm{~kg}^{-1}$ sand, $28 \mathrm{mg} \mathrm{kg}^{-1}$ available phosphorus (Olsen method), $690 \mathrm{mg} \mathrm{kg}^{-1}$ exchangeable potassium, $1.0 \mathrm{~g} \mathrm{~kg}^{-1}$ total $\mathrm{N}$ and $21 \mathrm{~g} \mathrm{~kg}^{-1}$ organic matter, $\mathrm{pH} 7.8$.

Under the CCS, plots received $76 \mathrm{~kg} \mathrm{ha}^{-1} \mathrm{P}_{2} \mathrm{O}_{5}$ at pre-seeding and $30 \mathrm{~kg} \mathrm{ha}^{-1} \mathrm{~N}$ applied at seedbed preparation as diammonium phosphate. Treatments with herbicides (Pendimethalin, $796 \mathrm{~g}$ a.i. $\mathrm{ha}^{-1}$ ) and fungicides (Azoxystrobin, $200 \mathrm{~g}$ a.i. ha- ${ }^{1}$ ) were applied according to local standard practices. The field trial included plots where the previous crop was durum wheat.

The soil preparation of the organic and conventional fields consisted of ploughing, hoeing and harrowing twice before sowing. The plots consisted of three 7.5-m long rows that were $50 \mathrm{~cm}$ apart (inter-row), which were sown with 50 seeds $\mathrm{m}^{-2}$ at the beginning of December (sowing at 12 December 2013 and at 12 December 2014, respectively, for the two growing seasons), under wet conditions. A marked difference in terms of weed pressure occurred between the two cropping systems, with higher values under the OCS experiments (Table S1, Supplementary Material).

\subsection{Weather Conditions}

Rainfall distribution and monthly mean maximum and minimum temperatures relative to 2014 and 2015 crop years are reported in Figure 1. The first year was wetter than the second one ( $445.6 \mathrm{~mm}$ in $2013 / 14$ vs. $237.6 \mathrm{~mm}$ in 2014/15), both in the early and late growing stages. A more comparable temperature distribution was observed between the two crop years, with a monthly mean of daily $\mathrm{T}^{\circ}$ max above $40^{\circ} \mathrm{C}$ in the late part of grain maturation in the first crop year (June 2014), which was generally slightly warmer.
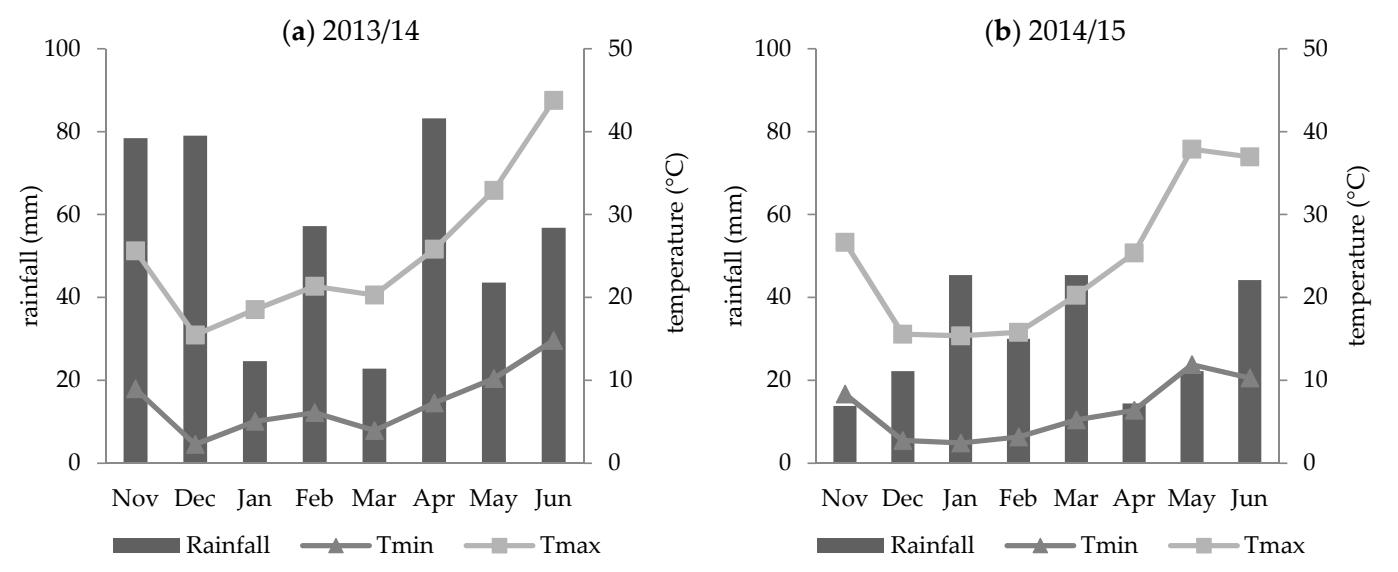

Figure 1. Monthly rainfall distribution and mean maximum and minimum temperatures in 2014 (a) and 2015 (b) crop years.

The two crop seasons differed in terms of biotic stress, with a markedly higher incidence of ascochyta blight (AB) in the wetter 2014 (10.0\% vs. 0.6\% of diseased plants, in 2014 and 2015, respectively).

\subsection{Agronomic Characterization and Seed Quality}

At maturity, seeds were harvested and grain yield (GY, in $\mathrm{kg} \mathrm{ha}^{-1}$ ) and seed weight (GW, in $\mathrm{mg}$ ) were determined and expressed on a dry matter basis. The number of seeds 
per $\mathrm{m}^{2}(\mathrm{GNO})$ was calculated by dividing GY by GW. Seed nitrogen $(\mathrm{N})$ concentration was measured with the Dumas combustion method (Leco FP528), and grain nitrogen uptake (GNU, in $\mathrm{kg}$ of $\mathrm{N} \mathrm{ha}^{-1}$ ) was calculated by multiplying GY to grain $\mathrm{N}$ concentration. Crude protein content $(\mathrm{PC})$ was determined by multiplying grain $\mathrm{N}$ concentration $\times 6.25$. Grains were ground in a Cyclotec 1093 mill (Tecator, Sweden) for chemistry analysis on proteins.

\subsection{Water-Holding Capacity}

Grain water-holding capacity (WHC) was determined according to a modified protocol adapted from [16]. Briefly, $5 \mathrm{~g}$ of flour (dry weight) was suspended in $50 \mathrm{~mL}$ of distilled water, mixed thoroughly for $30 \mathrm{~min}$ and then centrifuged at $5000 \mathrm{rpm}$ for $10 \mathrm{~min}$. The free water was removed from the wet flour which was then weighed. The average of two determinations was reported in grams of water per gram of flour.

\subsection{Protein Extraction and SDS-PAGE Analysis}

Soluble proteins were extracted from a protocol adapted from [17]. Briefly, $100 \mathrm{mg}$ of grounded flour was suspended with $1 \mathrm{~mL}$ of extraction buffer $(50 \mathrm{mM}$ Tris- $\mathrm{HCl}, \mathrm{pH} 7.8$, $5 \mathrm{mM}$ EDTA, 0.1\% 1,4-dithiothreitol) for $1 \mathrm{~h}$ at room temperature with constant mixing and centrifuged at 10,000 $\mathrm{g}$ for $30 \mathrm{~min}$. The supernatant, containing the total soluble proteins, was used to prepare samples for SDS-PAGE. The protein content in the supernatant was quantified according to the Bradford protocol. For each sample, $10 \mu \mathrm{L}$ of extracted proteins was separated by SDS-PAGE (at 12\%) using an SE 600 apparatus (Hoefer, Inc., Holliston, MA, USA). Gels were stained with Coomassie Brilliant Blue G250 and digitally acquired (Epson Perfection V750pro) [18]. Molecular weight markers, from 10 to $250 \mathrm{kDa}$, were used (Bio-Rad Co). Image analysis of gels was performed by ImageQuant TL software (Amersham Biosciences). The relative amount of each protein band abundance was determined by densitometric analysis and expressed as a percentage of the total protein amount in each gel lane. The expression of four groups of proteins was assessed on denaturated protein bands [19]: 7s convicilin ( $\sim 68-70 \mathrm{kDa}), 7 \mathrm{~S}$ vicilin $(\sim 43,50$ and $53 \mathrm{kDa}$ subunits), $11 \mathrm{~s}$ legumin ( $\sim 37$ and $\sim 25 \mathrm{kDa}$ as acid subunit $\alpha$ - and basic subunit $\beta$-, respectively), lectin $(\sim 32 \mathrm{kDa})$ and $2 \mathrm{~s}$ albumin $(\sim 11 \mathrm{kDa})$, and the ratio between $7 \mathrm{~s}$ vicilin and 11s legumin $(7 \mathrm{~s}-\mathrm{V} / 11 \mathrm{~s}-\mathrm{L})$ was then assessed.

\subsection{Statistical Analysis}

For each cropping system (OCS and CCS), the responses of the agronomic and quality parameters were subjected to a two-way (genotype, year, genotype $x$ year) analysis of the variance (ANOVA) and means were separated by Tukey's honestly significant difference (HSD, $p \leq 5 \%$ ). Furthermore, the means of the OCS and the CCS were statistically compared using Student's $t$-test, and percent change was reported according to this formula: $100 \times(\mathrm{OCS}-\mathrm{CCS}) / \mathrm{CCS}$. The Pearson correlation analysis between agronomic and quality parameters and protein composition was also carried out. Statistical analysis was performed by JMP software (Version 8.0.2, SAS Institute Inc., 2009).

\section{Results}

\subsection{Yield and Quality Response under Conventional and Organic Cropping Systems}

The analysis of the variance (ANOVA) generally showed, under both investigated cropping systems (CSs), a significant effect of genotype and of crop year on the investigated parameters (Table 2); their interaction, however, was not significant for grain yield (GY) and seed weight $(\mathrm{GW})$ and was generally not significant under the organic cropping system (OCS) for most of the agronomic parameters. A marked environmental effect on grain yield (GY) and yield components was observed between the two crop years. The climatic conditions occurring in the first crop year (2014), wetter and warmer than the second one, proved favorable for biotic pressure ( $\mathrm{AB}$ and weeds) and led to a general lower productivity under both cropping systems (Table 3), resulting in almost two times higher mean GY in the second crop year (1060 kg ha ${ }^{-1}$ in 2014 vs. $1995 \mathrm{~kg} \mathrm{ha}^{-1}$ in 2015 under the CCS; 
$525 \mathrm{~kg} \mathrm{ha}^{-1}$ in 2014 vs. $853 \mathrm{~kg} \mathrm{ha}^{-1}$ in 2015 under the OCS). These differences in GY were mainly associated with a higher number of seeds per $\mathrm{m}^{2}$ (GNO, 2657 vs. 4274), while seed weight (GW) was slightly significantly affected only under the CCS (Table 3). Furthermore, in 2015, a significantly higher number of seeds per $\mathrm{m}^{2}$ (GNO) and higher grain $\mathrm{N}$ uptake (GNU) were observed, more marked under the CCS (Table 3).

The investigated chickpea genotypes showed large variability in terms of agronomic performance under both the CCS and the OCS (Table 3). Vulcano showed the lowest GY while Nero Senise and Sultano were generally the most productive genotypes, with higher differences under the CCS with respect to the OCS. The same Nero Senise showed the lowest GW (234 mg under the CCS; $244 \mathrm{mg}$ under the OCS) while Reale and Pascià showed the highest values (Table 3). GNO and GNU showed the same behavior as GY, with the highest values showed by Nero Senise under the CCS and the OCS in both crop years (Table 4). The productivity results obtained under the two experimental cropping systems were markedly different, as reported in Table 5 . In particular, a marked yield gap ( $-58 \%)$ between the OCS and the CCS was observed, as for GNO and GNU ( $-56 \%)$. However, no difference was found in terms of GW (328 mg under the CCS; $344 \mathrm{mg}$ under the OCS), which was mainly affected by genotype. As for the investigated quality traits, the crop season showed a slight significant influence on protein concentration (Table 2), with a different trend between the two cropping systems-under the CCS, a lower general PC was found in the less productive year (2014); however, this effect was significant only for Pascià and Reale (Table 4). On the other hand, under the OCS, only Nero Senise showed a significantly higher PC in 2014 than in 2015, while the effect of the crop year was not significant for the other genotypes. The cultivar Pascià was characterized by the lowest mean PC $(22.3 \%$ under the CCS; $22.6 \%$ under the OCS), while the highest value was achieved by Principe $(25.2 \%)$, especially under organic management and in the wetter year $(27.7 \%)$. Indeed, for chickpea genotypes grown under Mediterranean rainfed conditions, PC generally turned out to be a stable trait within the ones investigated and was more influenced by genotypic variability rather than environmental effects. Indeed, no differences were observed between the two cropping systems (Table 5).

Table 2. Analysis of variance relative to the effect of genotype $(\mathrm{G})$, year $(\mathrm{Y})$ and of their interaction under the conventional cropping system (CCS) and the organic cropping system (OCS).

\begin{tabular}{|c|c|c|c|c|c|c|}
\hline \multirow[t]{2}{*}{ Parameter } & \multicolumn{3}{|c|}{ CCS } & \multicolumn{3}{|c|}{ OCS } \\
\hline & $\mathbf{G}$ & $\mathbf{Y}$ & $\mathbf{G} \times \mathbf{Y}$ & G & $\mathbf{Y}$ & $G \times Y$ \\
\hline GY & $* * *$ & $* * *$ & ns & * & $* * *$ & ns \\
\hline GW & $* * *$ & $* * *$ & ns & $*$ & ns & ns \\
\hline GNO & $* * *$ & $* * *$ & * & $* * *$ & $* * *$ & ns \\
\hline GNU & $* * *$ & $* * *$ & * & $* *$ & $* * *$ & ns \\
\hline PC & $*$ & $* * *$ & * & $*$ & $* * *$ & ns \\
\hline WHC & $* * *$ & $*$ & $*$ & $* * *$ & ns & $* *$ \\
\hline $7 s-C$ & $* * *$ & $*$ & $* * *$ & $* *$ & $* *$ & ns \\
\hline $7 s-V$ & $* * *$ & $* * *$ & $* * *$ & $* *$ & $* * *$ & $* * *$ \\
\hline $11 s-\mathrm{L}$ & $* * *$ & $* * *$ & $* * *$ & $* * *$ & $* * *$ & $* * *$ \\
\hline lect & $* * *$ & $* * *$ & $* * *$ & $* * *$ & ns & $* * *$ \\
\hline 2s-alb & $* * *$ & $* *$ & $*$ & ns & ns & ns \\
\hline 7s-V/11s-L & $* * *$ & $* * *$ & $* * *$ & $* * *$ & $* * *$ & $* * *$ \\
\hline
\end{tabular}

$\overline{\mathrm{GY}}=$ grain yield $; \mathrm{GW}=$ grain weight GNO = grain number; GNU = grain nitrogen uptake; PC = protein content $\mathrm{WHC}=$ water-holding capacity; $7 \mathrm{~s}-\mathrm{C}=$ convicilin; $7 \mathrm{~s}-\mathrm{V}=$ vicilin; $11 \mathrm{~s}-\mathrm{L}=$ legumin; lect $=$ lectin; $2 \mathrm{~s}-\mathrm{alb}=2 \mathrm{~s}$ albumin; $7 \mathrm{~s}-\mathrm{V} / 11 \mathrm{~s}-\mathrm{L}=$ ratio between $7 \mathrm{~s}$ vicilin and $11 \mathrm{~s}$ legumin. Level of significance: $\mathrm{ns}=$ not significant; ${ }^{*} p \leq 5 \%$; ** $p \leq 1 \%$; *** $p \leq 1 \%$. 


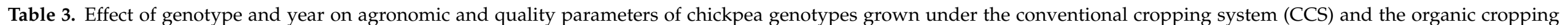
system (OCS).

\begin{tabular}{|c|c|c|c|c|c|c|c|c|c|c|c|c|c|c|}
\hline CS & Factor & Level & GY & GW & GNO & GNU & PC & WHC & $7 s-C$ & $7 s-V$ & $11 \mathrm{~s}-\mathrm{L}$ & lect & $2 s-a l b$ & $7 s-V / 11 s-L$ \\
\hline & & & $\mathrm{kg} \mathrm{ha}^{-1}$ & mg & no. $m^{-2}$ & $\mathrm{~kg} \mathrm{ha}^{-1}$ & $\%$ & $\mathrm{~g} \mathrm{~g}^{-1}$ & $\%$ & $\%$ & $\%$ & $\%$ & $\%$ & ratio \\
\hline \multirow[t]{9}{*}{ CCS } & Genotype & Calia & $1391 \mathrm{~b}-\mathrm{d}$ & $301 \mathrm{c}$ & $4507 \mathrm{bc}$ & $53.8 \mathrm{~b}-\mathrm{d}$ & $23.6 \mathrm{ab}$ & $1.47 \mathrm{~b}$ & $7.0 \mathrm{~b}-\mathrm{d}$ & $39.2 \mathrm{a}$ & $24.7 \mathrm{c}$ & $14.5 \mathrm{~b}$ & $5.9 \mathrm{bc}$ & $1.88 \mathrm{a}$ \\
\hline & & Kairo & $1717 \mathrm{a}-\mathrm{c}$ & $312 c$ & 5484 b & $65.8 \mathrm{ab}$ & $23.8 \mathrm{ab}$ & $1.34 \mathrm{~b}$ & $7.7 \mathrm{a}-\mathrm{c}$ & $39.3 \mathrm{a}$ & $24.0 \mathrm{c}$ & $15.8 \mathrm{a}$ & $5.0 \mathrm{c}$ & $1.97 \mathrm{a}$ \\
\hline & & Nero Senise & $2050 \mathrm{a}$ & $234 \mathrm{~d}$ & $8762 \mathrm{a}$ & 77.0 a & $23.6 \mathrm{ab}$ & $1.68 \mathrm{a}$ & $7.3 \mathrm{a}-\mathrm{d}$ & $24.5 \mathrm{~b}$ & $37.3 \mathrm{~b}$ & $10.7 \mathrm{e}$ & $7.5 \mathrm{a}$ & $0.85 \mathrm{~b}$ \\
\hline & & Pascià & $1361 \mathrm{~b}-\mathrm{d}$ & $382 \mathrm{~b}$ & $3403 c$ & $51.2 \mathrm{~b}-\mathrm{d}$ & $22.3 \mathrm{~b}$ & $1.33 \mathrm{~b}$ & $7.0 \mathrm{~b}-\mathrm{d}$ & $23.0 \mathrm{~b}$ & $37.3 \mathrm{~b}$ & $13.0 \mathrm{~d}$ & $5.2 \mathrm{c}$ & $0.81 \mathrm{bc}$ \\
\hline & & Principe & $1617 \mathrm{a}-\mathrm{d}$ & $378 \mathrm{~b}$ & 4243 bc & $63.5 \mathrm{a}-\mathrm{c}$ & $24.5 \mathrm{a}$ & $1.40 \mathrm{~b}$ & $6.2 \mathrm{~d}$ & $21.0 \mathrm{~d}$ & $39.3 \mathrm{a}$ & $14.3 \mathrm{bc}$ & $5.7 \mathrm{c}$ & $0.69 \mathrm{~d}$ \\
\hline & & Reale & $1176 \mathrm{~cd}$ & $429 a$ & $2715 c$ & $44.3 \mathrm{~cd}$ & $23.2 \mathrm{ab}$ & $1.39 \mathrm{~b}$ & $7.8 \mathrm{ab}$ & $24.2 \mathrm{~b}$ & $37.3 \mathrm{~b}$ & $12.7 \mathrm{~d}$ & $5.3 \mathrm{c}$ & $0.88 \mathrm{~b}$ \\
\hline & & Sultano & $1834 \mathrm{ab}$ & $306 \mathrm{c}$ & $5968 \mathrm{~b}$ & $69.7 \mathrm{ab}$ & $23.5 \mathrm{ab}$ & $1.42 \mathrm{~b}$ & $6.5 \mathrm{~cd}$ & $20.7 \mathrm{~d}$ & $40.8 \mathrm{a}$ & $11.0 \mathrm{e}$ & $7.2 \mathrm{ab}$ & $0.67 \mathrm{~d}$ \\
\hline & Year & 2014 & $1060 \mathrm{~b}$ & $310 \mathrm{~b}$ & $3645 b$ & $38.6 \mathrm{~b}$ & $22.7 \mathrm{~b}$ & $1.40 \mathrm{~b}$ & $7.0 \mathrm{~b}$ & $25.9 \mathrm{~b}$ & $36.8 \mathrm{a}$ & $12.5 \mathrm{~b}$ & $6.3 \mathrm{a}$ & $1.00 \mathrm{~b}$ \\
\hline & & 2015 & 1995 a & $347 a$ & $6010 \mathrm{a}$ & $78.3 \mathrm{a}$ & $24.6 \mathrm{a}$ & $1.47 \mathrm{a}$ & $7.5 \mathrm{a}$ & $27.2 \mathrm{a}$ & $33.4 \mathrm{~b}$ & $13.9 \mathrm{a}$ & $5.6 \mathrm{~b}$ & $1.12 \mathrm{a}$ \\
\hline \multirow[t]{8}{*}{ OCS } & Genotype & Calia & $573 \mathrm{bc}$ & $355 a-c$ & 1661 c & $21.0 \mathrm{bc}$ & $23.2 \mathrm{~b}$ & $1.38 \mathrm{~b}$ & $7.3 \mathrm{a}$ & $42.3 \mathrm{ab}$ & $23.2 \mathrm{~d}$ & $12.5 \mathrm{ab}$ & $7.0 \mathrm{a}$ & $2.15 \mathrm{a}$ \\
\hline & & Kairo & $714 \mathrm{a}-\mathrm{c}$ & $321 b-d$ & $2196 \mathrm{a}-\mathrm{c}$ & $26.8 \mathrm{a}-\mathrm{c}$ & $24.1 \mathrm{ab}$ & $1.39 \mathrm{~b}$ & $6.3 \mathrm{~b}$ & $41.5 \mathrm{a}-\mathrm{c}$ & $26.5 \mathrm{a}$ & $12.7 \mathrm{ab}$ & $6.2 \mathrm{a}$ & $1.82 \mathrm{c}$ \\
\hline & & Nero Senise & $802 \mathrm{ab}$ & $244 \mathrm{~d}$ & $3288 \mathrm{a}$ & $30.0 \mathrm{ab}$ & $23.7 \mathrm{ab}$ & $1.60 \mathrm{a}$ & $6.2 \mathrm{~b}$ & $42.7 \mathrm{a}$ & $24.3 \mathrm{~b}-\mathrm{d}$ & $10.3 \mathrm{~d}$ & $7.2 \mathrm{a}$ & $2.01 \mathrm{ab}$ \\
\hline & & Pascià & $649 a-c$ & $432 \mathrm{a}$ & 1505 c & $23.2 \mathrm{a}-\mathrm{c}$ & $22.6 \mathrm{~b}$ & $1.34 \mathrm{~b}$ & $7.0 \mathrm{ab}$ & $39.7 \mathrm{c}$ & $27.0 \mathrm{a}$ & $13.3 \mathrm{a}$ & $6.5 \mathrm{a}$ & $1.77 \mathrm{c}$ \\
\hline & & Principe & $763 a-c$ & $370 a-c$ & 2061 bc & $31.3 \mathrm{ab}$ & $25.8 \mathrm{a}$ & $1.32 \mathrm{~b}$ & $6.3 \mathrm{~b}$ & $40.0 \mathrm{bc}$ & $26.2 \mathrm{ab}$ & $11.0 \mathrm{~cd}$ & $6.8 \mathrm{a}$ & $1.78 \mathrm{c}$ \\
\hline & & Reale & $627 \mathrm{a}-\mathrm{c}$ & $400 \mathrm{ab}$ & 1700 c & $23.5 a-c$ & $23.3 \mathrm{ab}$ & $1.34 \mathrm{~b}$ & $6.5 \mathrm{ab}$ & $43.0 \mathrm{a}$ & $24.5 \mathrm{~b}-\mathrm{d}$ & $11.0 \mathrm{~cd}$ & $6.7 \mathrm{a}$ & $2.03 \mathrm{ab}$ \\
\hline & Year & 2014 & $525 \mathrm{~b}$ & $338 a$ & $1670 \mathrm{~b}$ & $21.0 \mathrm{~b}$ & $24.9 \mathrm{a}$ & $1.41 \mathrm{a}$ & $6.3 \mathrm{~b}$ & $39.8 \mathrm{~b}$ & $26.5 \mathrm{a}$ & $11.8 \mathrm{a}$ & $6.4 \mathrm{a}$ & $1.76 \mathrm{~b}$ \\
\hline & & 2015 & 853 a & $350 \mathrm{a}$ & 2538 a & $30.8 \mathrm{a}$ & $22.5 \mathrm{~b}$ & $1.38 \mathrm{a}$ & $6.9 \mathrm{a}$ & $42.8 \mathrm{a}$ & $23.7 \mathrm{~b}$ & $11.7 \mathrm{a}$ & $6.9 \mathrm{a}$ & $2.10 \mathrm{a}$ \\
\hline
\end{tabular}

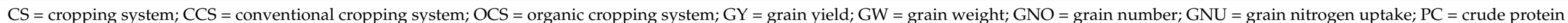

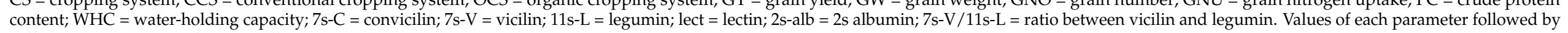
different letters are significantly different according to Tukey's test. 
Table 4. Effect of the interaction genotype (G) x year (Y) of chickpea varieties grown under the conventional cropping system (CCS) and the organic cropping system (OCS).

\begin{tabular}{|c|c|c|c|c|c|c|c|c|c|c|c|c|c|c|c|c|c|c|}
\hline \multirow{3}{*}{$\begin{array}{c}\text { Genotype } \\
\text { CCS }\end{array}$} & \multirow{2}{*}{\multicolumn{2}{|c|}{$\begin{array}{c}\text { GNO } \\
\text { n. ha }{ }^{-1}\end{array}$}} & \multirow{2}{*}{\multicolumn{2}{|c|}{$\begin{array}{c}\text { GNU } \\
\text { kg ha }^{-1}\end{array}$}} & \multirow{2}{*}{\multicolumn{2}{|c|}{$\begin{array}{c}\text { PC } \\
\%\end{array}$}} & \multirow{2}{*}{\multicolumn{2}{|c|}{$\begin{array}{l}\text { WHC } \\
\mathrm{g} \mathrm{g}^{-1}\end{array}$}} & \multirow{2}{*}{\multicolumn{2}{|c|}{$\begin{array}{c}\text { 7s conv } \\
\% \\
\end{array}$}} & \multirow{2}{*}{\multicolumn{2}{|c|}{$7 \mathrm{~s}$ vicil }} & \multicolumn{2}{|c|}{ 11s leg } & \multicolumn{2}{|c|}{ lect } & \multicolumn{2}{|c|}{$2 \mathrm{~s}$ alb } \\
\hline & & & & & & & & & & & & & & & & & & \\
\hline & 2014 & 2015 & 2014 & 2015 & 2014 & 2015 & 2014 & 2015 & 2014 & 2015 & 2014 & 2015 & 2014 & 2015 & 2014 & 2015 & 2014 & 2015 \\
\hline Calia & 2934 e-h & $6080 a-d$ & $29.0 \mathrm{e}-\mathrm{g}$ & $78.7 \mathrm{a}-\mathrm{d}$ & $22.1 \mathrm{a}-\mathrm{c}$ & $25.1 \mathrm{a}$ & $1.45 \mathrm{a}-\mathrm{d}$ & $1.48 \mathrm{a}-\mathrm{d}$ & $6.7 \mathrm{c}$ & $7.3 \mathrm{bc}$ & $37.0 \mathrm{~b}$ & $41.3 \mathrm{a}$ & $26.0 \mathrm{f}$ & $23.3 \mathrm{fg}$ & $15.7 \mathrm{a}$ & $13.3 \mathrm{~cd}$ & $6.9 \mathrm{ab}$ & $5.0 \mathrm{~b}-\mathrm{d}$ \\
\hline Kairo & $4373 c-g$ & $6595 \mathrm{a}-\mathrm{c}$ & $49.0 \mathrm{c}-\mathrm{f}$ & $82.7 \mathrm{ab}$ & $22.9 \mathrm{a}-\mathrm{c}$ & $24.7 \mathrm{ab}$ & $1.19 \mathrm{~d}$ & $1.48 \mathrm{a}-\mathrm{d}$ & $7.7 \mathrm{a}-\mathrm{c}$ & $7.7 \mathrm{a}-\mathrm{c}$ & $41.7 \mathrm{a}$ & $37.0 \mathrm{~b}$ & $23.0 \mathrm{~g}$ & $25.0 \mathrm{fg}$ & $15.3 \mathrm{ab}$ & $16.3 \mathrm{a}$ & $5.0 \mathrm{~b}-\mathrm{d}$ & $5.0 \mathrm{~b}-\mathrm{d}$ \\
\hline $\begin{array}{l}\text { Nero } \\
\text { Senise }\end{array}$ & $8676 \mathrm{a}$ & $8848 \mathrm{a}$ & $68.7 \mathrm{a}-\mathrm{d}$ & $85.3 \mathrm{ab}$ & $23.6 \mathrm{ab}$ & $23.5 \mathrm{ab}$ & $1.72 \mathrm{a}$ & $1.64 \mathrm{ab}$ & $7.7 \mathrm{a}-\mathrm{c}$ & $7.0 \mathrm{bc}$ & $24.0 \mathrm{~d}$ & $25.0 \mathrm{~d}$ & $38.7 \mathrm{~b}-\mathrm{d}$ & 36.0 de & $8.0 \mathrm{~g}$ & $13.3 \mathrm{~cd}$ & $7.4 \mathrm{a}$ & $7.5 \mathrm{a}$ \\
\hline Pascià & $1928 \mathrm{gh}$ & $4877 \mathrm{~b}-\mathrm{f}$ & $22.7 \mathrm{fg}$ & $79.7 \mathrm{a}-\mathrm{c}$ & $20.0 \mathrm{c}$ & $24.5 \mathrm{ab}$ & $1.28 \mathrm{~cd}$ & $1.39 \mathrm{a}-\mathrm{d}$ & $7.0 \mathrm{bc}$ & $7.0 \mathrm{bc}$ & $22.0 \mathrm{e}$ & $24.0 \mathrm{~d}$ & $38.7 \mathrm{~b}-\mathrm{d}$ & 36.0 de & $12.0 \mathrm{de}$ & $14.0 \mathrm{bc}$ & $6.0 \mathrm{a}-\mathrm{d}$ & $4.5 \mathrm{~cd}$ \\
\hline Principe & $3462 \mathrm{~d}-\mathrm{h}$ & $5023 \mathrm{~b}-\mathrm{f}$ & $47.3 \mathrm{~d}-\mathrm{f}$ & $79.7 \mathrm{a}-\mathrm{c}$ & $24.1 \mathrm{ab}$ & $25.0 \mathrm{a}$ & $1.32 \mathrm{~b}-\mathrm{d}$ & $1.49 \mathrm{a}-\mathrm{d}$ & $6.3 c$ & $6.0 \mathrm{c}$ & 20.7 ef & $21.3 \mathrm{ef}$ & $40.7 \mathrm{bc}$ & $38.0 \mathrm{~cd}$ & $12.3 \mathrm{de}$ & $16.3 \mathrm{a}$ & $6.5 \mathrm{a}-\mathrm{c}$ & $5.0 \mathrm{~b}-\mathrm{d}$ \\
\hline Reale & $2090 \mathrm{f}-\mathrm{h}$ & $3339 \mathrm{~d}-\mathrm{h}$ & $30.0 \mathrm{e}-\mathrm{g}$ & $58.7 \mathrm{~b}-\mathrm{e}$ & $21.6 \mathrm{bc}$ & $24.9 \mathrm{a}$ & $1.49 \mathrm{a}-\mathrm{d}$ & $1.28 \mathrm{~cd}$ & $6.7 c$ & $9.0 \mathrm{a}$ & $21.3 \mathrm{ef}$ & $27.0 \mathrm{c}$ & $41.0 \mathrm{~b}$ & $33.7 \mathrm{e}$ & $11.3 \mathrm{ef}$ & $14.0 \mathrm{bc}$ & $6.5 a-c$ & $4.0 \mathrm{~d}$ \\
\hline Sultano & $4307 \mathrm{c}-\mathrm{h}$ & $7629 \mathrm{ab}$ & $47.0 \mathrm{~d}-\mathrm{g}$ & $92.3 \mathrm{a}$ & $22.7 \mathrm{a}-\mathrm{c}$ & $24.4 \mathrm{ab}$ & $1.38 \mathrm{~b}-\mathrm{d}$ & $1.45 \mathrm{a}-\mathrm{d}$ & $6.7 \mathrm{c}$ & $6.3 c$ & $20.7 \mathrm{ef}$ & $20.7 \mathrm{ef}$ & $41.3 \mathrm{~b}$ & $40.3 \mathrm{bc}$ & $12.0 \mathrm{de}$ & $10.0 \mathrm{f}$ & $6.5 \mathrm{a}-\mathrm{c}$ & $8.0 \mathrm{a}$ \\
\hline Vulcano & $1388 \mathrm{~h}$ & $5690 \mathrm{~b}-\mathrm{e}$ & $15.3 \mathrm{~g}^{\circ}$ & $69.7 \mathrm{a}-\mathrm{d}$ & $24.8 \mathrm{a}$ & $24.8 \mathrm{a}$ & $1.38 \mathrm{~b}-\mathrm{d}$ & $1.53 \mathrm{a}-\mathrm{c}$ & $8.0 \mathrm{a}-\mathrm{c}$ & $9.0 \mathrm{a}$ & $20.0 \mathrm{f}$ & $21.3 \mathrm{ef}$ & $44.7 \mathrm{a}$ & $35.0 \mathrm{e}$ & $13.0 \mathrm{~cd}$ & $14.0 \mathrm{bc}$ & $6.0 \mathrm{a}-\mathrm{d}$ & $6.0 \mathrm{a}-\mathrm{d}$ \\
\hline OCS & 2014 & 2015 & 2014 & 2015 & 2014 & 2015 & 2014 & 2015 & 2014 & 2015 & 2014 & 2015 & 2014 & 2015 & 2014 & 2015 & 2014 & 2015 \\
\hline Calia & $1055 \mathrm{~d}$ & $2267 \mathrm{a}-\mathrm{d}$ & $13.3 \mathrm{~d}$ & $28.7 \mathrm{a}-\mathrm{d}$ & $23.7 \mathrm{a}-\mathrm{c}$ & $22.7 \mathrm{bc}$ & $1.45 \mathrm{~b}-\mathrm{d}$ & $1.32 \mathrm{c}-\mathrm{e}$ & $7.3 \mathrm{a}$ & $7.3 \mathrm{a}$ & $43.0 \mathrm{a}-\mathrm{c}$ & $41.7 \mathrm{a}-\mathrm{c}$ & $22.7 \mathrm{c}$ & $23.7 \mathrm{c}$ & $13.3 \mathrm{bc}$ & $11.7 \mathrm{c}-\mathrm{f}$ & $7.5 \mathrm{a}$ & $6.5 \mathrm{a}$ \\
\hline Kairo & $1291 \mathrm{~cd}$ & $3101 \mathrm{a}-\mathrm{c}$ & $16.3 \mathrm{~cd}$ & $37.3 \mathrm{ab}$ & $25.5 \mathrm{a}-\mathrm{c}$ & $22.7 \mathrm{bc}$ & $1.30 \mathrm{c}-\mathrm{e}$ & $1.47 \mathrm{bc}$ & $6.0 \mathrm{a}$ & $6.7 \mathrm{a}$ & $40.0 \mathrm{~b}-\mathrm{d}$ & $43.0 \mathrm{a}-\mathrm{c}$ & $28.0 \mathrm{ab}$ & $25.0 \mathrm{bc}$ & $10.3 \mathrm{fg}$ & $15.0 \mathrm{ab}$ & $6.9 \mathrm{a}$ & $5.6 \mathrm{a}$ \\
\hline $\begin{array}{l}\text { Nero } \\
\text { Senise }\end{array}$ & $3218 \mathrm{ab}$ & $3359 \mathrm{ab}$ & $30.7 \mathrm{a}-\mathrm{d}$ & $29.3 \mathrm{a}-\mathrm{d}$ & $25.8 \mathrm{ab}$ & $21.7 \mathrm{c}$ & $1.68 \mathrm{a}$ & $1.52 \mathrm{ab}$ & $6.3 \mathrm{a}$ & $6.0 \mathrm{a}$ & $42.3 \mathrm{a}-\mathrm{c}$ & $43.0 \mathrm{a}-\mathrm{c}$ & $25.0 \mathrm{bc}$ & $23.7 \mathrm{c}$ & $11.3 \mathrm{~d}-\mathrm{f}$ & $9.3 \mathrm{~g}$ & $5.5 \mathrm{a}$ & $9.0 \mathrm{a}$ \\
\hline Pascià & $1064 \mathrm{~d}$ & 1947 a-d & $17.0 \mathrm{~cd}$ & $29.3 \mathrm{a}-\mathrm{d}$ & $23.2 \mathrm{bc}$ & $21.9 \mathrm{bc}$ & $1.39 \mathrm{~b}-\mathrm{e}$ & $1.29 \mathrm{de}$ & $7.0 \mathrm{a}$ & $7.0 \mathrm{a}$ & $34.3 \mathrm{e}$ & $45.0 \mathrm{a}$ & $30.0 \mathrm{a}$ & $24.0 \mathrm{c}$ & $15.3 \mathrm{a}$ & $11.3 \mathrm{~d}-\mathrm{f}$ & $5.6 \mathrm{a}$ & $7.5 \mathrm{a}$ \\
\hline Principe & $1922 \mathrm{a}-\mathrm{d}$ & $2200 \mathrm{a}-\mathrm{d}$ & $29.7 \mathrm{a}-\mathrm{d}$ & $33.0 \mathrm{a}-\mathrm{c}$ & $27.7 \mathrm{a}$ & $23.8 \mathrm{a}-\mathrm{c}$ & $1.39 \mathrm{~b}-\mathrm{e}$ & $1.27 \mathrm{e}$ & $6.0 \mathrm{a}$ & $6.7 \mathrm{a}$ & $39.3 \mathrm{~cd}$ & $40.7 \mathrm{~b}-\mathrm{d}$ & $28.0 \mathrm{ab}$ & $24.3 \mathrm{c}$ & $10.7 \mathrm{e}-\mathrm{g}$ & $11.3 \mathrm{~d}-\mathrm{f}$ & $6.6 \mathrm{a}$ & $7.1 \mathrm{a}$ \\
\hline Reale & $1559 \mathrm{~b}-\mathrm{d}$ & $1841 \mathrm{a}-\mathrm{d}$ & $22.0 \mathrm{~b}-\mathrm{d}$ & $25.0 \mathrm{a}-\mathrm{d}$ & $24.3 \mathrm{a}-\mathrm{c}$ & $22.4 \mathrm{bc}$ & $1.32 \mathrm{c}-\mathrm{e}$ & $1.36 \mathrm{~b}-\mathrm{e}$ & $6.0 \mathrm{a}$ & $7.0 \mathrm{a}$ & $42.3 \mathrm{a}-\mathrm{c}$ & $43.7 \mathrm{ab}$ & $25.7 \mathrm{bc}$ & $23.3 \mathrm{c}$ & $10.3 \mathrm{fg}$ & $11.7 \mathrm{c}-\mathrm{f}$ & $6.5 \mathrm{a}$ & $6.8 \mathrm{a}$ \\
\hline Sultano & $2179 \mathrm{a}-\mathrm{d}$ & $3542 \mathrm{a}$ & $26.0 \mathrm{a}-\mathrm{d}$ & $41.3 \mathrm{a}$ & $24.0 \mathrm{a}-\mathrm{c}$ & $22.8 \mathrm{bc}$ & $1.43 \mathrm{~b}-\mathrm{e}$ & $1.41 \mathrm{~b}-\mathrm{e}$ & $6.0 \mathrm{a}$ & $7.0 \mathrm{a}$ & $40.0 \mathrm{~b}-\mathrm{d}$ & $42.7 \mathrm{a}-\mathrm{c}$ & $25.0 \mathrm{bc}$ & $23.0 \mathrm{c}$ & $12.3 \mathrm{c}-\mathrm{e}$ & $10.3 \mathrm{ef}$ & $6.0 \mathrm{a}$ & $6.5 \mathrm{a}$ \\
\hline Vulcano & $1073 \mathrm{~d}$ & $2048 \mathrm{a}-\mathrm{d}$ & $13.3 \mathrm{~d}$ & $22.7 \mathrm{a}-\mathrm{d}$ & $24.6 \mathrm{a}-\mathrm{c}$ & $22.1 \mathrm{~b}-\mathrm{c}$ & $1.33 \mathrm{c}-\mathrm{e}$ & $1.42 \mathrm{~b}-\mathrm{e}$ & $6.0 \mathrm{a}$ & $7.3 \mathrm{a}$ & 37.0 de & $43.0 \mathrm{a}-\mathrm{c}$ & $28.0 \mathrm{ab}$ & $22.7 \mathrm{c}$ & $11.0 \mathrm{f}-\mathrm{g}$ & $13.0 \mathrm{~cd}$ & $7.0 \mathrm{a}$ & $6.5 \mathrm{a}$ \\
\hline
\end{tabular}

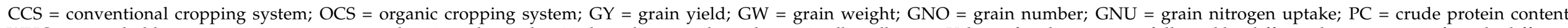

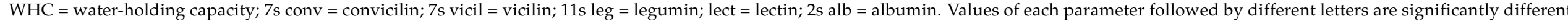
according to Tukey's test. 
Table 5. Mean changes of agronomic and quality parameters between the conventional cropping system (CCS) and the organic cropping systems (OCS).

\begin{tabular}{ccccc}
\hline Parameter & Unit & CCS & OCS & OCS vs. CCS \\
\hline GY & $\mathrm{kg} \mathrm{ha}^{-1}$ & $1527 \pm 93$ & $689 \pm 37$ & $-58 \%$ \\
GW & $\mathrm{mg}^{-2}$ & $328 \pm 9.4$ & $344 \pm 9.5$ & $5 \%$ \\
GNO & $\mathrm{no.} \mathrm{m}^{-2}$ & $4827 \pm 346$ & $2104 \pm 137$ & $-56 \%$ \\
GNU & $\mathrm{kg} \mathrm{ha}^{-1}$ & $58.5 \pm 3.8$ & $25.9 \pm 1.3$ & $-56 \%$ \\
PC & $\%$ & $23.7 \pm 0.3$ & $23.7 \pm 0.2$ & $0 \%$ \\
WHC & $\mathrm{g} \mathrm{g}^{-1}$ & $1.43 \pm 0.02$ & $1.40 \pm 0.02$ & $-2 \%$ \\
7s convicilin & $\%$ & $7.3 \pm 0.2$ & $6.6 \pm 0.1$ & $-10 \%$ \\
7s vicilin & $\%$ & $26.6 \pm 1.1$ & $41.3 \pm 0.4$ & $55 \%$ \\
11s legumin & $\%$ & $35.1 \pm 0.7$ & $25.1 \pm 0.2$ & $-28 \%$ \\
lectin & $\%$ & $13.2 \pm 2.2$ & $11.8 \pm 1.7$ & $-11 \%$ \\
2s albumin & $\%$ & $6.0 \pm 0.3$ & $6.7 \pm 0.2$ & $12 \%$ \\
7s-V/11s-L & ratio & $1.06 \pm 0.2$ & $1.93 \pm 0.1$ & $82 \%$ \\
\hline
\end{tabular}

$\mathrm{GY}=$ grain yield; $\mathrm{GW}=$ grain weight; $\mathrm{GNO}=$ grain number; $\mathrm{GNU}=$ grain nitrogen uptake; $\mathrm{PC}=$ crude protein content; $\mathrm{WHC}=$ water-holding capacity; $7 \mathrm{~s}-\mathrm{V} / 11 \mathrm{~s}-\mathrm{L}=$ ratio between $7 \mathrm{~s}$ vicilin and $11 \mathrm{~s}$ legumin. For each parameter, mean values and standard errors are reported.

As for the physicochemical properties of flour, the effect of genotype generally prevailed over the other ones (Table 2). The mean effect of crop year was significant only under the CCS (Table 3); however, the changes between 2014 and 2015 were not significant for any genotype (Table 4). An interesting genotypic variability was observed with black-seed Nero Senise, showing the highest water-holding capacity (WHC) in all the investigated agronomic and environmental conditions. Values observed in the two crop years were comparable and ranged from 1.19 to $1.72 \mathrm{~g}$ water retained per $\mathrm{g}$ of flour. No differences between the mean values of the two cropping systems (CCS vs. OCS) were observed (Table 5).

\subsection{Changes in Chickpea Protein Composition}

SDS-PAGE allowed to discriminate the relative expression of the globulin-like (7s convicilin, $7 \mathrm{~s}$ vicilin and 11s legumin) and albumin-like (lectin and $2 \mathrm{~s}$ albumin) fractions in the different chickpea genotypes grown under the organic (ORG) and conventional (CONV) farming systems. A higher prevalence of globulin-like proteins was observed for all genotypes under all the agronomic and environmental conditions. Vicilin and legumin were the most abundant fractions, followed by lectin, convicilin and $2 \mathrm{~s}$ albumin, as shown in Table 4. The effect of the environment (year), under both the CCS and the OCS, resulted in a slightly higher legumin abundance in the less productive 2014, while the other fractions showed an opposite trend (Table 3).

A differential response of the chickpea genotypes was observed in relation to the expression of 7s vicilin and 11-S legumin under the two contrasting cropping systems (Table 3). In particular, a higher vicilin content was observed under the OCS with respect to the CCS $(+55 \%)$; indeed, under the CCS, all genotypes showed values ranging between $20.0 \%$ and $25.0 \%$, except for Calia and Kairo, which showed a significantly higher expression $(39.2 \%$, Table 3$)$. On the other hand, a higher mean range occurred under the OCS $(34.4-43.0 \%)$. The $11 \mathrm{~s}$ legumin fraction generally showed an opposite behavior with respect to $7 \mathrm{~s}$ vicilin, with a $-28 \%$ mean decrease under organic management with respect to the conventional one (Table 5). On average, Calia showed the lowest legumin expression, under both cropping systems (Table 4).

The proportion of the two main fractions was also evaluated and expressed in terms of vicilin-to-legumin ratio (7s-V/11s-L), as reported in Figure 2. Except for Calia and Kairo, under the CCS, values of the ratio were generally below 1.0; on the other hand, $7 \mathrm{~s}-\mathrm{V} / 11 \mathrm{~s}-\mathrm{L}$ was markedly higher under the OCS (+82\%, Table 5). 


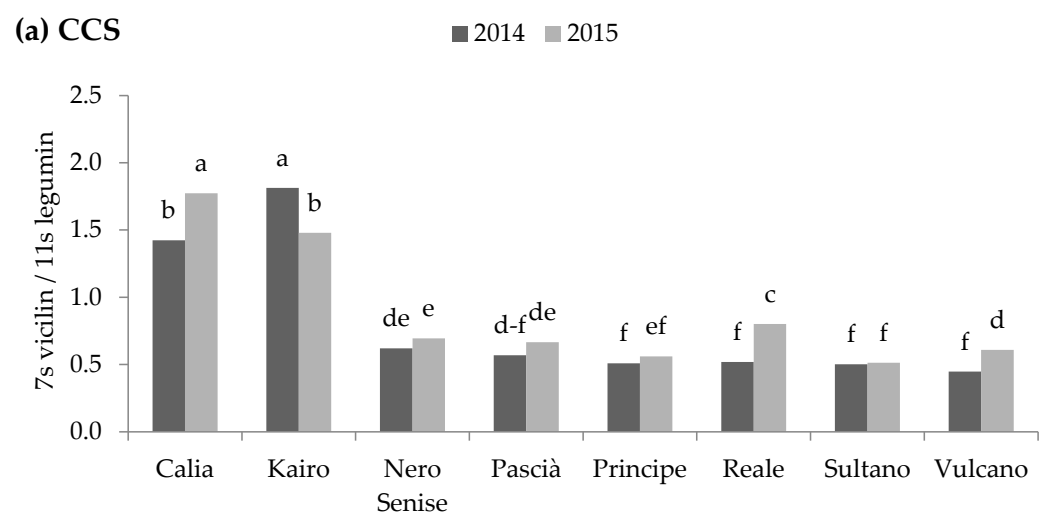

(b) OCS $\quad \square 2014 \square 2015$

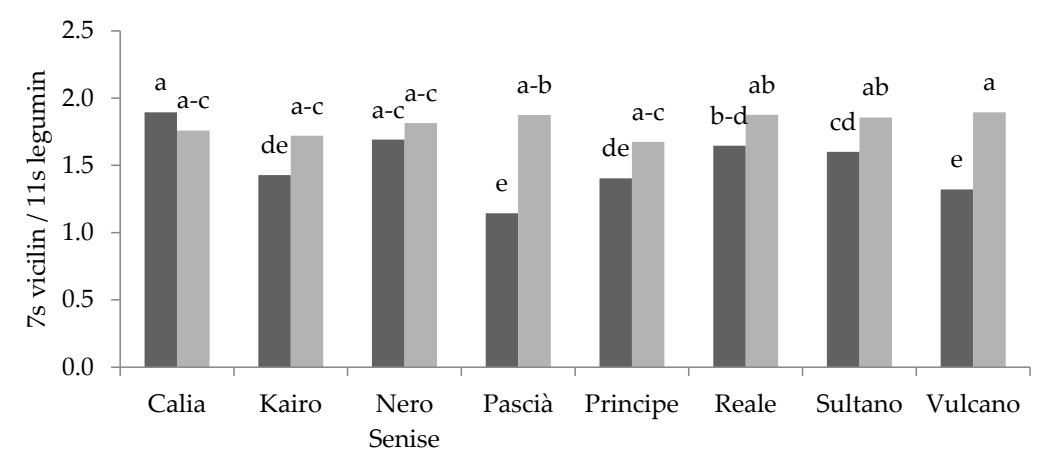

Figure 2. Ratio between $7 \mathrm{~s}$ vicilin and $11 \mathrm{~s}$ legumin expression $(7 \mathrm{~s}-\mathrm{V} / 11 \mathrm{~s}-\mathrm{L})$ in eight chickpea genotypes grown in a two-year field trial (2014, black; 2015, gray) under conventional (CCS, (a)) and organic (OCS, (b)) cropping systems. In each cropping system, values of each parameter followed by different letters are significantly different according to Tukey's test.

As for lectin relative abundance, on average, Nero Senise showed the lowest values under both cropping systems. Furthermore, a higher range was observed under the CCS with respect to the OCS (8.0-16.3\% CCS vs. 9.3-15.0\% OCS, as reported in Table 4), with higher mean values (Table 4). A comparable trend was observed for $7 \mathrm{~s}$ convicilin that, under the CCS, ranged from $6.0 \%$ to $9.0 \%$, with higher mean values for Vulcano, while under the OCS, a more reduced range (6.0-7.3\%) was observed (Table 4), with no genotype showing changes between crop years. On average, a slightly lower content was observed under the OCS $(-10 \%$ relative to the CCS, Table 4$)$.

As for the low molecular weight $2 \mathrm{~s}$ albumin, different mean values were observed between crop years only under the CCS (6.4\% in 2014 vs. 5.6\% in 2015); under the OCS, no significant differences were observed between genotypes in interaction with crop year, and mean values were slightly higher $(+12 \%)$ than from the CCS (Table 4$)$.

\subsection{Relationship between Chickpea Protein Composition and Agronomic Traits}

The results of the multiple regression analysis between agronomic and quality traits are reported in Table 6. GY, which strongly correlated with GNO and GNU rather than with GW, also showed a positive correlation with legumin and a negative correlation with vicilin and the 7s-V/11s-L ratio. PC, which did not show marked variability, showed no significant correlation with the expression of any identified protein fractions; however, a negative trend with $2 \mathrm{~s}$ albumin was observed. As for WHC, a positive correlation was observed with PC while negative with GW and 7s-V/11s-L ratio. 
Table 6. Matrix of correlation between agronomic parameters, protein content and composition.

\begin{tabular}{|c|c|c|c|c|c|c|c|c|c|c|c|}
\hline & GW & GNO & GNU & PC & WHC & $7 \mathrm{~s}-\mathrm{C}$ & $7 s-V$ & 11s-L & lectin & $2 s-a l b$ & $7 \mathrm{~s}-\mathrm{V} / 11 \mathrm{~s}-\mathrm{L}$ \\
\hline GY & -0.10 & 0.93 & 0.99 & 0.13 & 0.31 & 0.25 & -0.45 & 0.33 & 0.22 & -0.14 & -0.39 \\
\hline GW & & -0.38 & -0.10 & -0.10 & -0.41 & 0.07 & 0.02 & -0.04 & 0.23 & -0.24 & 0.02 \\
\hline GNO & & & 0.92 & 0.10 & 0.46 & 0.22 & -0.41 & 0.31 & 0.06 & 0.00 & -0.35 \\
\hline GNU & & & & 0.22 & 0.33 & 0.25 & -0.45 & 0.38 & 0.23 & -0.16 & -0.39 \\
\hline PC & & & & & 0.25 & 0.02 & -0.03 & 0.04 & 0.00 & -0.20 & -0.07 \\
\hline WHC & & & & & & -0.04 & -0.15 & 0.15 & -0.09 & 0.06 & -0.25 \\
\hline $7 s-C$ & & & & & & & -0.16 & 0.07 & 0.29 & -0.22 & -0.06 \\
\hline $7 s-V$ & & & & & & & & -0.96 & -0.08 & 0.09 & 0.98 \\
\hline $11 \mathrm{~s}-\mathrm{L}$ & & & & & & & & & -0.04 & -0.06 & -0.98 \\
\hline lectin & & & & & & & & & & -0.42 & -0.02 \\
\hline $2 \mathrm{~s}-\mathrm{alb}$ & & & & & & & & & & & 0.09 \\
\hline
\end{tabular}

$\mathrm{GY}=$ grain yield $\mathrm{GW}=$ grain weight; $\mathrm{GNO}=$ grain number; $\mathrm{GNU}=$ grain nitrogen uptake; $\mathrm{PC}=$ protein content; WHC = water-holding capacity; $7 \mathrm{~s}-\mathrm{C}=7 \mathrm{~s}$ convicilin; $7 \mathrm{~s}-\mathrm{V}=7 \mathrm{~s}$ vicilin; $11 \mathrm{~s}-\mathrm{L}=11 \mathrm{~s}$ legumin; $2 \mathrm{~s}-\mathrm{alb}=2 \mathrm{~s}$ albumin; $7 \mathrm{~s}-\mathrm{V} / 11 \mathrm{~s}-\mathrm{L}=$ ratio between $7 \mathrm{~s}$ vicilin to $11 \mathrm{~s}$ legumin. Bold numbers indicate $\mathrm{R}$ values with level of significance at $p \leq 5 \%$.

\section{Discussion}

The results from the field trials showed a marked influence of genetic variability and environment and suggested an effect of crop management on grain yield and its components. The highest productivity achieved by chickpea genotypes, under lower rainfall conditions, is in agreement with a previous study in Mediterranean environments where the authors observed that the highest seed yield was obtained with about $390 \mathrm{~mm}$ of rainfall and that wetter conditions led to a decrease in crop production [20]. The authors suggested that excessive rainfall may led to a negative effect due to waterlogging on chickpea plants. Similar results were obtained in the same environment with different irrigation regimes: irrigation application greater than $170 \mathrm{~mm}$ decreased chickpea GY and water use efficiency as well due to ascochyta blight (AB) infection [21]. Furthermore, in another study on chickpeas grown under Mediterranean conditions [6], the authors stated that the lower production under wetter conditions might also be ascribable to higher biotic stress pressure. Under our experimental conditions, the higher rainfall was associated to a higher weed pressure that competed for nutrient availability and $A B$ that limited the crop development. Insufficient crop soil nutrient availability and the effects of diseases and pests are generally associated to a significantly low grain yield under organic management [22]. Indeed, conditions of optimal humidity and temperature during reproductive stages can be favorable for $\mathrm{AB}$, with possible loss of production [23]; this helps to explain the large gap between CCS and OCS that occurred in the wettest year. The organic farming condition suffered more from the wetter weather of the first year, which stimulated weed growth and, thus, limited nutrients uptake and the radiation interception of chickpeas.

A wide genetic variability was observed in terms of grain productivity within the chickpea genotypes. The best agronomic performances are generally obtained by the genotypes with the highest adaptability [8]. In a previous study conducted under Mediterranean conditions, the cultivar Sultano showed a higher GY with respect to Pascià [6]. Those differences might be also ascribable to genetic $\mathrm{AB}$ resistance under specific favorable conditions. Furthermore, under our experimental conditions, the cultivar Sultano showed an interesting suitability for organic farming, as previously suggested [6], followed by Nero Senise. The use of suitable genetic resources may have a key role in promoting sustainable crop production, including pulses under low-input organic farming systems.

A reduced variability was observed in relation to PC between CCS and OCS [22], which showed an effect of management only under better environmental conditions. The higher PC observed under organic farming in the first crop season was inversely related to GY, with a consequent $\mathrm{N}$ concentration effect in grains [24]. Under conventional management, however, the highest production in the second crop year was consistent with the highest PC. This was possibly due to the generally better physiological response of the crop that, under less limiting conditions with respect to organic farming (weed 
competition), took advantage of the highest $\mathrm{N}$ amount, ordinarily applied before sowing in conventional management on chickpea [6]. This practice is recommended within crop rotations with cereals, taking into account the reduced $\mathrm{N}$ fixation that generally occurs with chickpea compared to other pulses [25]. Indeed, $\mathrm{N}$ uptake in chickpea is strongly influenced by $\mathrm{N}$ supply in soil, which, as a consequence, regulates final grain yield [26].

Despite a moderate genetic effect on protein concentration, a large variability in terms of protein composition was observed in the current study. In recent years, the evidence of an existing genetic diversity in terms of seed protein composition was exploited in cultivated pulses, including chickpeas $[8,17,27,28]$. Most of the information on pulse protein composition was obtained by electrophoretic separation and is relative to genetic diversity $[8,29,30]$, while less data are available on the effect of environmental [31] or agronomic factors [32]. In our study, the interaction of genotypic factor under different crop managements (organic vs. conventional) showed a marked significant variability. The predominance of $7 \mathrm{~s}$ vicilin and 11s legumin is in accordance with the literature on pulse storage proteins $[3,27,33]$. Both proteins are classified on the basis of the sedimentation coefficient, and they are generally oligomeric proteins, trimers for $7 \mathrm{~s}$ vicilin and hexamer for 11s legumin [3]. A large variability exists in literature in terms of the prevalence of the two proteins. In a study conducted on pea genotypes, a higher proportion of vicilin than legumin was observed, in a range from 1.6 to 8.2 [27]; furthermore, in a second experiment conducted on pea, a higher legumin prevalence over vicilin was found [32]. The values observed in the current investigation (0.63-2.23) are in line with the ranges reported in the literature. In our study, vicilin and legumin were mainly responsible for changes in protein composition and were also found to be strongly influenced by environmental and agronomic factors. The existence of a significant variability in terms of the proportion of $7 \mathrm{~s}$ and 11s globulins was already observed in pea [32] in relation to both environment (crop season and crop site) and management (seed density). The authors observed a negative relationship between $7 \mathrm{~s}-\mathrm{V} / 11 \mathrm{~s}-\mathrm{L}$ and protein content; this trend was in accordance with ours in the most productive crop year, and this ratio was generally negatively associated to grain yield. In addition, different soil mineral availability can also affect chickpea seed composition [34].

Changes in chickpea protein composition may have relevance both for technological [28] and health aspects [35]. Besides the direct consumption of chickpea as food, there is also an increasing interest in the use of chickpea flour as a protein additive in cereal flour [36] or as gluten-free flour [37] and also for sustainable goals [38]. Different physicochemical tests are adopted to estimate the technological performances of a food matrix. Among these, the water-holding capacity is the ability of a protein matrix to absorb and physically retain water against gravity by bound, hydrodynamic and capillary interactions [39]; indeed, fiber may also contribute to increase WHC. The values observed in the current study are in the range comprised within the literature for pulses [40], with the higher values observed in the black and small seeds being in accordance with previous findings [10]. The genetic variability found may be a useful basis for industrial application, in particular for chickpea flour consumption and also in addition to cereal flours with different technological attitudes [41]. The differences in SDS-PAGE profiling may help to explain the rheological properties of the flours in order to individuate the major protein fractions responsible for technological performance in pulses [16]. The variation observed in water absorption capacity in chickpea cultivars is suggested to be due to differences in hydrophilic groups in protein concentrates [29].

As for health aspects, four peptides (ALEPDHR, TETWNPNHPEL, FVPH and SAEHGSLH) obtained from hydrolysate chickpea legumin after digestion have recently been proposed for their antioxidant properties [42]; in addition, the 50-kDa subunit of vicilin and the 20-kDa basic subunit of legumin were reported as putative chickpea allergens [43]. The reduced convicilin (7s globulin) content is in accordance with [27], which also found no correlation with the amount of vicilin and legumin in pea. 
The reduced lectin variability was associated to lower values in the more productive conventional management. Legume lectins, with their rich hydrophobic amino acid regions that allow interactions with other molecules, are known for their antinutritional properties [44], in particular their hemagglutinating capacity. However, chickpea lectin may depress starch digestion, leading to a lower glycemic index [45]. An interesting genotypic variability was found in our study with lower content in the black-seed genotype Nero Senise, which showed high adaptability and good productivity in the investigated Mediterranean environment.

The $2 \mathrm{~s}$ albumin, which consists of two subunits, generally accounts for $\sim 10 \%$ of legume storage proteins, in accordance with the values observed in our study, but $\sim 50 \%$ of total sulfur content, as cysteine amino acid [7]. Although under our experimental conditions, it showed few changes, Nero Senise showed a higher content than other genotypes, suggesting a possible implication in terms of amino acid composition with more sulfur-rich ones.

The observed changes in protein composition suggest an important effect of crop management, in particular organic farming, associated to genetic differences in terms of crop and quality response. These changes seem related to agronomic traits and technological performances and can be further explored by a proteomic approach.

\section{Conclusions}

The results of this study suggest how organic farming has a marked impact not only on crop productivity but also on chickpea protein composition. The observed genotypic differences also indicate the presence of a promising genetic variability in terms of suitability to organic farming, possibly due to a higher resistance to abiotic and biotic stresses. The cultivars Sultano and black-seed Nero Senise emerged as the most productive, also under organic farming. Crop year and agronomic management showed a dramatic impact on grain yield and its components. In addition, this was associated more with changes in protein composition rather than content. In particular, a negative correlation between grain yield and the ratio of $7 \mathrm{~s}$ vicilin with $11 \mathrm{~s}$ legumin proteins was observed. The role of these proteins is also related to an allergenic potential of some sub-fractions; for this reason, information on the impact of environment and crop management on their expression could be relevant for health aspects. Minor changes were observed in terms of water-holding capacity, a physical parameter relevant for technological performance, mostly related to genetic variability. Chickpea flours with high values might be used to enhance the technological value of cereal flour mixtures. Furthermore, an interesting genotypic variability was also observed in terms of lectin expression, a protein associated to antinutritional properties, with a trend of lower values under organic farming.

In conclusion, the present study highlights the importance of the choice of genotypes for cultivation under organic farming and how this can impact not only crop productivity but also technological and health quality, in chickpea. Further proteomic studies are needed to support these results, under contrasting environmental and management conditions.

Supplementary Materials: The following are available online at https: / www.mdpi.com/2073-4 395/11/2/191/s1, Table S1: Mean of weed plants (number $\mathrm{m}^{-2}$ ) in conventional (CCS) and organic (OCS) cropping systems on chickpea sampled on 2014 and 2015 at Foggia. Table S2: Mean of protein subunit expression of eight chickpea genotypes grown under two contrasting cropping systems (CCS vs OCS).

Author Contributions: Conceptualization, M.A.D.S., M.R., C.F. and Z.F.; methodology, M.A.D.S., M.R. and C.F.; formal analysis, M.A.D.S.; investigation, M.A.D.S., V.M., P.C. and L.G.; writingoriginal draft preparation, M.A.D.S.; writing—review and editing, M.R., C.F. and Z.F.; visualization, M.A.D.S.; supervision, C.F. and Z.F.; project administration, M.R. and C.F.; funding acquisition, Z.F. All authors have read and agreed to the published version of the manuscript.

Funding: Michele Andrea De Santis is the beneficiary of a Grant by MIUR in the framework of “AIM: Attraction and International Mobility" (PON R\&I2014-2020) (D74I18000180001). 
Institutional Review Board Statement: Not applicable.

Informed Consent Statement: Not applicable.

Data Availability Statement: Data sharing not applicable.

Acknowledgments: The authors thank Maria Grazia Cataldi for her technical support.

Conflicts of Interest: The authors declare no conflict of interest.

\section{References}

1. Ghelfi, R.; Palmieri, A. Pulses production in Italy: Trade, marketing and policy issues. Ital. J. Agron. 2017, 12, 891. [CrossRef]

2. Liu, K.; Bandara, M.; Hamel, C.; Knight, J.D.; Gan, Y. Intensifying crop rotations with pulse crops enhances system productivity and soil organic carbon in semi-arid environments. Field Crops Res. 2020, 248, 107657. [CrossRef]

3. Duranti, M. Grain legume proteins and nutraceutical properties. Fitoterapia 2006, 77, 67-82. [CrossRef] [PubMed]

4. Maiti, R.K.; Wesche-Ebeling, P. Vegetative and reproductive growth and productivity. In Advances in Chickpea Science; Maiti, R., Wesche-Ebeling, P., Eds.; Science Publishers: Enfield, NH, USA, 2001; pp. 67-104.

5. Nisa, Z.U.; Arif, A.; Waheed, M.Q.; Shah, T.M.; Iqbal, A.; Siddiqui, A.J.; Choudhary, M.I.; El-Seedi, H.R.; Musharraf, S.G. A comparative metabolomic study on desi and kabuli chickpea (Cicer arietinum L.) genotypes under rainfed and irrigated field conditions. Sci. Rep. 2020, 10, 13919. [CrossRef] [PubMed]

6. Ruggeri, R.; Primi, R.; Danieli, P.P.; Ronchi, B.; Rossini, F. Effects of seeding date and seeding rate on yield, proximate composition and total tannins content of two Kabuli chickpea cultivars. Ital. J. Agron. 2017, 12, 890. [CrossRef]

7. Shewry, P.R. The protein chemistry of dicotyledonous grains. Encyclopedia Food Grains 2016, 2, 109-114. [CrossRef]

8. Zaccardelli, M.; Sonnante, G.; Lupo, F.; Piergiovanni, A.R.; Laghetti, G.; Sparvoli, F.; Lioi, L. Characterization of Italian chickpea (Cicer arietinum L.) germplasm by multidisciplinary approach. Genet. Resour. Crop Evol. 2013, 60, 865-877. [CrossRef]

9. De Giovanni, C.; Pavan, S.; Taranto, F.; Di Rienzo, V.; Miazzi, M.M.; Marcotrigiano, A.R.; Mangini, G.; Montemurro, C.; Ricciardi, L.; Lotti, C. Genetic variation of a global germplasm collection of chickpea (Cicer arietinum L.) including Italian accessions at risk of genetic erosion. Physiol. Mol. Biol. Plants 2017, 23, 197-205. [CrossRef]

10. Summo, C.; De Angelis, D.; Ricciardi, L.; Caponio, F.; Lotti, C.; Pavan, S.; Pasqualone, A. Nutritional, physico-chemical and functional characterization of a global chickpea collection. J. Food Compos. Anal. 2019, 43, 49-60. [CrossRef]

11. Vadez, V.; Berger, J.D.; Warkentin, T.; Asseng, S.; Ratnakumar, P.; Rao, K.P.C.; Gaur, P.M.; Munier-Jolain, N.; Larmure, A.; Voisin, A.S.; et al. Adaptation of grain legumes to climate change: A review. Agron. Sustain. Dev. 2012, 32, 31-34. [CrossRef]

12. Kaloki, P.; Trethowan, R.; Tan, D.K.Y. Genetic and environmental influences on chickpea water-use efficiency. J. Agron. Crop Sci. 2019, 205, 470-476. [CrossRef]

13. Dubey, R.K.; Dubey, P.K.; Chaurasia, R.; Singh, H.B.; Abhilash, P.C. Sustainable agronomic practices for enhancing the soil quality and yield of Cicer arietinum L. under diverse agroecosystems. J. Environ. Manag. 2020, 262, 110284. [CrossRef] [PubMed]

14. Menga, V.; Codianni, P.; Fares, C. Agronomic Management under Organic Farming May Affect the Bioactive Compounds of Lentil (Lens culinaris L.) and Grass Pea (Lathyrus communis L.)? Sustainability 2014, 6, 1059-1075. [CrossRef]

15. Ksiezak, J.; Bojarszczuk, J. The Effect of Cropping Method and Botanical Form on Seed Yielding and Chemical Composition of Chickpeas (Cicer arietinum L.) Grown under Organic System. Agronomy 2020, 10, 801. [CrossRef]

16. Ravi, R.; Harte, J.B. Milling and physicochemical properties of chickpea (Cicer arietinum L.) varieties. J. Sci. Food Agric. 2009, 89, 258-266. [CrossRef]

17. Nikolic, Z.; Dordevic, V.; Torbica, A.; Mikic, A. Legumes seed storage proteins characterization by SDS-PAGE and Lab-on-a-Chip electrophoresis. J. Food Compos. Anal. 2012, 28, 75-80. [CrossRef]

18. De Santis, M.A.; Giuliani, M.M.; Flagella, Z.; Reyneri, A.; Blandino, M. Impact of nitrogen fertilisation strategies on the protein content, gluten composition and rheological properties of wheat for biscuit production. Field Crops Res. 2020, 254. [CrossRef]

19. Ladjal-Ettoumi, Y.; Boudries, H.; Chibane, M.; Romero, A. Pea, chickpea and lentil protein isolates: Physicochemical characterization and emulsifying properties. Food Biophys. 2016, 11, 43-51. [CrossRef]

20. López-Bellido, F.J.L.; López-Bellido, R.J.; Khalil, S.K.; López-Bellido, L.L. Effect of planting date on winter kabuli chickpea growth and yield under rainfed Mediterranean conditions. Agron. J. 2008, 100, 957-964. [CrossRef]

21. Rinaldi, M.; Vonella, A.V.; Soldo, P.; Debiase, G.; Garofalo, P. Yield and canopy response of chickpea (Cicer arietinum L.) to different irrigation regimes. In Sustainable Irrigation-Management, Technologies and Policies; Villacampa Esteve, Y., Brebbia, C.A., Pratas Rico, D., Eds.; WIT Press, WIT Transaction on Ecology and the Environment: Southampton, UK, 2008; Volume 112, pp. 123-132. [CrossRef]

22. Caliskan, S.; Erdogan, C.; Arslan, M.; Caliskan, M.E. Comparison of organic and traditional production systems in chickpea (Cicer arietinum L.). Turk. J. Field Crops 2013, 18, 34-39.

23. Zhang, C.; Chen, W.; Sankaran, S. Impact of ascochyta blight disease on the expression of biochemical compounds in chickpea. Crop Prot. 2019, 125, 104885. [CrossRef]

24. Gaur, P.M.; Singh, M.K.; Samineni, S.; Sajja, S.B.; Jukanti, A.K.; Kamatam, S.; Varshney, R.K. Inheritance of protein content and its relationships with seed size, grain yield and other traits in chickpea. Euphytica 2016, 209, 253-260. [CrossRef] 
25. López-Bellido, R.J.; López-Bellido, L.; Benítez-Vega, J.; Munoz-Romero, V.; López-Bellido, F.; Redondo, R. Chickpea and faba bean nitrogen fixation in a Mediterranean rainfed Vertisol: Effect of the tillage system. Europ. J. Agron. 2011, 34, 222-230. [CrossRef]

26. López-Bellido, R.J.; López-Bellido, L.; Castillo, J.E.; López-Bellido, F.J. Chickpea response to tillage and soil residual nitrogen in a continuous rotation with wheat II. Soil nitrate, N uptake and influence on wheat yield. Field Crops Res. 2004, 88, 201-210. [CrossRef]

27. Tzitzikas, E.N.; Vincken, J.P.; De Groot, J.; Gruppen, H.; Visser, R.G.F. Genetic variation in pea seed globulin composition. J. Agric. Food Chem. 2006, 54, 425-433. [CrossRef]

28. Lioi, L.; Sparvoli, F.; Sonnante, G.; Laghetti, G.; Lupo, F.; Zaccardelli, M. Characterization of Italian grasspea (Lathyrus sativus L.) germplasm using agronomic traits, biochemical and molecular markers. Genet. Resour. Crop Evol. 2011, 58, 425-437. [CrossRef]

29. Singh, G.D.; Wani, A.A.; Kaur, D.; Sogi, D.S. Characterisation and functional properties of proteins of some Indian chickpea (Cicer arietinum) cultivars. J. Sci. Food Agric. 2008, 88, 778-786. [CrossRef]

30. Barac, M.; Cabrilo, S.; Pesic, M.; Stanojevic, S.; Zilic, S.; Macej, O.; Ristic, N. Profile and functional properties of seed proteins from six pea (Pisum sativum) genotypes. Int. J. Mol. Sci. 2010, 11, 4973-4990. [CrossRef]

31. Arefian, M.; Vessal, S.; Malekzadeh-Shafaroudi, S.; Siddique, H.M.; Bagheri, A. Comparative proteomics and gene expression analyses revealed responsive proteins and mechanisms for salt tolerance in chickpea genotypes. BMC Plant Biol. 2019, 19, 300. [CrossRef]

32. Mertens, C.; Dehon, L.; Bourgeois, A.; Verhaeghe-Cartrysse, C.; Blecker, C. Agronomical factors influencing the legumin/vicilin ratio in pea (Pisum sativum L.) seeds. J. Sci. Food Agric. 2012, 92, 1591-1596. [CrossRef]

33. Tavano, O.L.; Neves, V.A. Isolation, solubility and in vitro hydrolysis of chickpea vicilin-like protein. LWT Food Sci. Technol. 2008, 41, 1244-1251. [CrossRef]

34. Chiaiese, P.; Ohkama-Ohtsu, N.; Molvig, L.; Godfree, R.; Dove, H.; Hocart, C.; Fujiwara, T.; Higgins, T.J.V.; Tabe, L.M. Sulphur and nitrogen nutrition influence the response of chickpea seeds to an added, transgenic sink for organic sulphur. J. Exper. Bot. 2004, 55, 404. [CrossRef]

35. Gupta, R.K.; Gupta, K.; Sharma, A.; Das, M.; Ansari, I.A.; Dwivedi, P.D. Health risks and benefits of chickpea (Cicer arietinum) consumption. J. Agric. Food Chem. 2017, 65, 6-22. [CrossRef] [PubMed]

36. Osorio-Diaz, P.; Agama-Acevedo, E.; Mendoza-Vinalay, M.; Tovar, J.; Bello-Pèrez, L.A. Pasta added with chickpea flour: Chemical composition, in vitro starch digestibility and predicted glycemic index. Cienc. Tecnol. Aliment. 2008, 6, 6-12. [CrossRef]

37. Sofi, S.A.; Singh, J.; Muzaffar, K.; Majid, D.; Dar, B.N. Physicochemical characteristics of protein isolates from native and germinated chickpea cultivars and their noodle quality. Int. J. Gastron. Food Sci. 2020, 22, 100258. [CrossRef]

38. Saget, S.; Costa, M.; Barilli, E.; de Vasconcelos, M.W.; Sancho Santos, C.; Styles, D.; Williams, M. Substituting wheat with chickpea flour in pasta production delivers more nutrition at a lower environmental cost. Sustain. Prod. Consum. 2020, 24, 26-38. [CrossRef]

39. Traynham, T.L.; Myers, D.J.; Carriquiry, A.L.; Johnson, L.A. Evaluation of water-holding capacity for wheat-soy flour blends. J. Am. Oil Chem. Soc. 2007, 84, 151-155. [CrossRef]

40. Du, S.; Jiang, H.; Yu, X.; Jane, J. Physicochemical and functional properties of whole legume flour. Food Sci. Technol. 2014, 308-313. [CrossRef]

41. Pasqualone, A.; De Angelis, D.; Squeo, G.; Difonzo, G.; Caponio, F.; Summo, C. The effect of the addition of Apulian black chickpea flour on the nutritional and qualitative properties of durum wheat-based bakery products. Foods 2019, 8, 504. [CrossRef]

42. Ghribi, A.M.; Sila, A.; Przybylski, R.; Nedjar-Arroume, N.; Makhlouf, I.; Blecker, C.; Besbes, S. Purification and identification of novel antioxidant peptides from enzymatic hydrolysate of chickpea (Cicer arietinum L.) protein concentrate. J. Funct. Foods 2015, 12, 516-525. [CrossRef]

43. Bar-El Dadon, S.; Pascual, C.Y.; Eshel, D.; Teper-Bamnolker, P.; Ibańez, M.D.P.; Reifen, R. Vicilin and the basic subunit of legumin are putative CP allergens. Food Chem. 2013, 138, 13-18. [CrossRef] [PubMed]

44. Gautam, A.J.; Gupta, N.; Narvekar, D.T.; Bhadkariya, R.; Bhagyawant, S.S. Characterization of chickpea (Cicer arietinum L.) lectin for biological activity. Physiol. Mol. Biol. Plants 2018, 24, 389-397. [CrossRef] [PubMed]

45. Prathapan, A.; Fahad, K.; Thomas, B.K.; Philip, R.M.; Raghu, K.G. Effect of sprouting on antioxidant and inhibitory potential of two varieties of Bengal gram (Cicer arietinum L.) against key enzymes linked to type-2 diabetes. Int. J. Food Sci. Nutr. 2011, 62, 234-238. [CrossRef] [PubMed] 